\title{
METRIC DOCUMENTATION OF CULTURAL HERITAGE: RESEARCH DIRECTIONS FROM THE ITALIAN GAMHER PROJECT
}

\author{
G. Bitelli ${ }^{\mathrm{a}}$, C. Balletti ${ }^{\mathrm{b}}$, R. Brumana ${ }^{\mathrm{c}}$, L. Barazzettic ${ }^{\mathrm{c}}$, M.G. D’Urso ${ }^{\mathrm{d}}$, F. Rinaudo ${ }^{\mathrm{e}}$, G. Tucci $^{\mathrm{f}}$ \\ ${ }^{\text {a }}$ Department of Civil, Chemical, Environmental and Materials Engineering (DICAM) \\ Alma Mater Studiorum - University of Bologna, Viale Risorgimento 2, 40136, Bologna \\ gabriele.bitelli@unibo.it \\ b Dipartimento di Architettura Costruzione Conservazione \\ Università Iuav di Venezia, Santa Croce 191, 30135, Venezia \\ balletti@iuav.it \\ ${ }^{\mathrm{c}}$ Department of Architecture, Built environment and Construction engineering (ABC) \\ Politecnico di Milano, Via Ponzio 31, 20133 Milano \\ (raffaella.brumana, luigi.barazzetti)@polimi.it \\ d Department of Civil and Mechanical Engineering (DICeM), University of Cassino and Southern Lazio \\ via G. Di Biasio, 43, 03043, Cassino \\ durso@unicas.it \\ e Dipartimento di Architettura e Design, Politecnico di Torino \\ Viale Mattioli, 39, 10125 Torino \\ fulvio.rinaudo@polito.it \\ ${ }^{\mathrm{f}}$ Geomatics for Environment and Conservation of Cultural Heritage Laboratory \\ University of Florence, Via Pier Antonio Micheli, 8, 50121 Florence \\ grazia.tucci@unifi.it
}

Commission II

KEY WORDS: CH documentation, Building Information Model (BIM), Geomatics, Photogrammetry

\begin{abstract}
:
GAMHer is a collaborative project that aims at exploiting and validating Geomatics algorithms, methodologies and procedures in the framework of new European regulations, which require a more extensive and productive use of digital information, as requested by the Digital Agenda for Europe as one of the seven pillars of the Europe 2020 Strategy. To this aim, GAMHer focuses on the need of a certified accuracy for surveying and monitoring projects with photogrammetry and laser scanning technologies, especially when used in a multiscale approach for landscape and built heritage documentation, conservation, and management.

The approach used follows a multi-LoD (level of detail) transition that exploits GIS systems at the landscape scale, BIM technology and "point cloud based" 3d modelling for the scale of the building, and an innovative BIM/GIS integrated approach to foster innovation, promote users' collaboration and encourage communication between users. The outcomes of GAMHer are not intended to be used only by a community of Geomatics specialists, but also by a heterogeneous user community that exploit images and laser scans in their professional activities.
\end{abstract}

\section{INTRODUCTION}

The way we use photogrammetry and laser scanning is going to have a larger impact according to the European Union Public Procurement Directive (EUPPD), which is strictly connected to the requirement of the Digital Agenda for Europe. Wider use of digital data has to be achieved, in Europe as well as internationally.

Geomatics information is directly affect by the requirements of the Directive and, at the same time, it assumes a fundamental importance with an increasing demand of innovative methodologies to collect, process, validate and exchange digital information for simplifying the public procurement regime. In this sense, a multiresolution approach based on surveying techniques able to produce 3D models offers the robust connection expected at different levels and for different specialists.

Photogrammetry and laser scanning have been successfully used in several applications requiring accurate digital reconstructions. The opportunity to follow a scalable approach, starting from the environmental level to the local one, makes Geomatics a unique source of information in projects for landscape and built heritage knowledge, conservation, valorization and management.

It is well known that digital data, including cartographic data and information at the level of the building (local level), can be captured with sensors mounted on satellites, airplanes, UAV platforms, and from the ground level. Using mainly photogrammetric and laser scanning methods, raw observations are turned into digital products for the different specialists involved in the project. This has a direct connection to the Digital Agenda for Europe, which promotes the use of digital technologies to stimulate Europe's economy.

In this context, Built Heritage constitutes a specific field to be investigated for its peculiarities respect to the new constructions. A reliable digital 3D model, if rigorously built, can be used in different fields of application: from BIM to FEM, from documentation towards conservation to dissemination of our heritage. Usable virtual copies or physical replicas can be employed for real and virtual restoration, or for visual and tactile exploration of objects. These models constitute a new approach that can join metric data, semantic information and object 
attributes towards a collaborative project, as also stated by EUPPD.

The change of scale, from the building to the landscape level, can be furthermore seen as a progressive approach towards Geographic Information System (GIS) based information. Today, 3D models, particularly BIM and GIS, are often used as separated tools, notwithstanding their common base consisting in spatial information with georeferenced entities described by attributes. BIM-GIS integration is not only expected, but mandatory to ensure a convergence of multidisciplinary interests. Here, methods for data collection and processing are the main source of information for the generation of reliable digital 3D models. This is a fundamental aspect, especially for the increasing availability of low-cost software on the commercial market, sometimes able only to provide "crude" reconstructions without metric integrity, that do not match the rigorousness of classical photogrammetric methods. Indeed, the new processing algorithms developed in recent years can significantly automate the different phases of the $3 \mathrm{D}$ reconstruction pipeline. On the other hand, this improved automation comes at a cost, which is a scarce attention to the reliability of the output, which cannot be neglected in the case of real metric application. Methods to evaluate the quality of the output must therefore be developed to validate if the achieved output has certified accuracy, demonstrating if it can be useful for productive work in different fields of application.

This paper describes the project GAMHer (Geomatics data Acquisition and Management for landscape and built Heritage in an European perspective), which is a 3 year project financed under the Italian PRIN 2015 framework (Progetti di Ricerca di Rilevante Interesse Nazionale). GAMHer will tackle the previous issues in the framework of the new European directives (UEPPD) supporting an increasing demand of innovative methodologies to collect, process, validate and exchange digital information. Particular attention will be paid to the realization of tools and guidelines for a data acquisition/processing workflow of images and laser scans towards a reliable and accurate output for real, cost-effective and productive work. GAMHer will investigate the advantages and limitations of actual image and laser scan processing algorithms and procedures with both a technologyand application-driven purpose, i.e. the combined need of automation and reliability. This can improve the overall production pipeline for the generation of metric digital reconstruction, fulfilling metric requisites while preserving automation.

The approach will follow a multiscale transition that exploits GIS systems at the landscape scale, BIM technology and "point cloud based" 3D modelling (including pure geometric modeling) for the scale of small artefacts, buildings, and infrastructures, and an innovative BIM/GIS integrated approach to foster innovation, promote users' collaboration and encourage communication between specialists and non-specialists.

\section{THE GAMHER PROJECT: STRUCTURE AND OVERALL OBJECTIVES}

\subsection{Overview}

Starting from the European research framework, managed through the HORIZON2020 action, the Italian Ministry of Research founded a special national call aiming also to support Italian researchers in developing basic research activities in view of the European calls. One of the winners is the GAMHer project (Geomatics data Acquisition and Management for landscape and built Heritage in a European perspective). The project, under the University di Bologna coordination, links other 5 Italian research groups mainly involved in $\mathrm{CH}$ documentation: Politecnico di Milano, Politecnico di Torino, IUAV Venice, University of Florence and University of Cassino and Southern Lazio. The GAMHer project aims to face a set of research and practical challenges in those disciplines devoted to generate 3D models for objects related to both landscape and built heritage. Therefore, natural and urban landscapes, buildings, and monuments are the main subjects of the project.

Different aspects were considered while designing the project, such as the growing request for an improved management and a wider fruition at the level of built cultural heritage and landscape. This has a strong impact on the acquisition, processing and management of (geospatial) data.

According to the GAMHer's concept, issues can be observed from two different points of views:

- at the level of professionals in charge for the maintenance, preservation and promotion of built heritage;

- $\quad$ at the level of information for citizens, exploiting their direct involvement for a better comprehension and social sharing of Cultural Heritage.

The overall activity has been structured in three main tasks (Fig. 1) that are directly linked to the two mentioned levels with the integration of specific problems devoted to the analysis of technical issues.

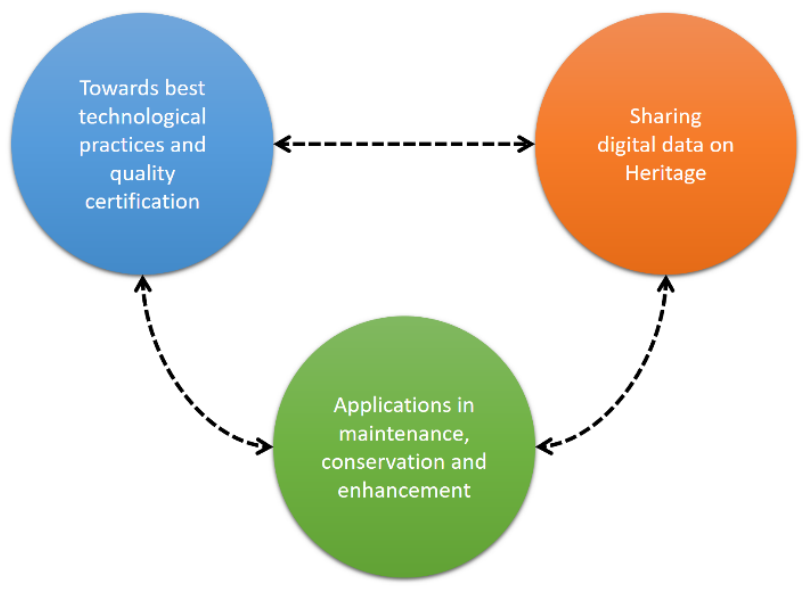

Figure 1. The three interconnected tasks that constitute GAMHer's tasks.

Such tasks were developed and linked starting from different considerations. First, recent technological progress has led to a reduction of the gap between the professionals and the amateurs. This aspect can be declined not only for the acquisition devices, but also for the realization of the final products, as far as acquisition and processing of data in an economic and transferable way. Modern photogrammetric systems are particularly interesting for the production of 3D models, with available online services for their elaboration, visualization and solid 3D printing. Spreading a technology from niche sectors to a significant number of "expert users", and hence to a mass audience, exhibits interesting opportunities but shows also some critical points that must be considered. It is a path similar to that, which has transformed the traditional cartographic production towards "neocartography" and "crowdmapping".

An interesting context for the Cultural Heritage concerns the spread and sharing of geospatial data between not expert users, i.e. non-specialist users that could be directly involved in digital documentation and mapping. Nowadays, a direct implication of such users is surely more practicable and technically feasible. It 
can bring undeniable social benefits in terms of sensibility and awareness on the knowledge and the protection of its own territory, as well as in the generation of wider databases, in contrast with the lack of resources currently dedicated to preservation and promotion of cultural heritage.

The need to realize comparative evaluations between the most modern 3D surveying techniques for the cultural heritage and, more generally, for the built environment, is transversal and common between the above mentioned contexts. Scanning methodologies and, above all, the photogrammetric ones, will be used by themselves or mutually integrated. Aim of the project is to verify, under several conditions and interpretations, the quality of the algorithmic approaches and techniques available today, in both scientific and commercial products, privileging whenever possible open software solutions since they are more easily transferable. The theoretical and practical comparison between the traditional photogrammetry and SfM techniques (Hartley and Zisserman, 2003) is, undoubtedly, one of the themes more interesting and critic for a wider spread of these techniques.

\subsection{GAMHer's main objectives}

GAMHer will provide instruments and guidelines designed considering not only technology-driven possibilities, but also realistic user-driven necessities. The increasing demand for accurate multilevel geoinformation is pushing the interest in the direct spotlight of the potentials of available technologies, with a particular attention at local and regional levels, where databases and georeferenced data are used for the promotion, conservation, valorization, safeguard and management of the built environment.

The impact of GAMHer (Figure 2) focuses in providing innovative services that would tackle major research needs on most relevant topics in the geospatial data sector.

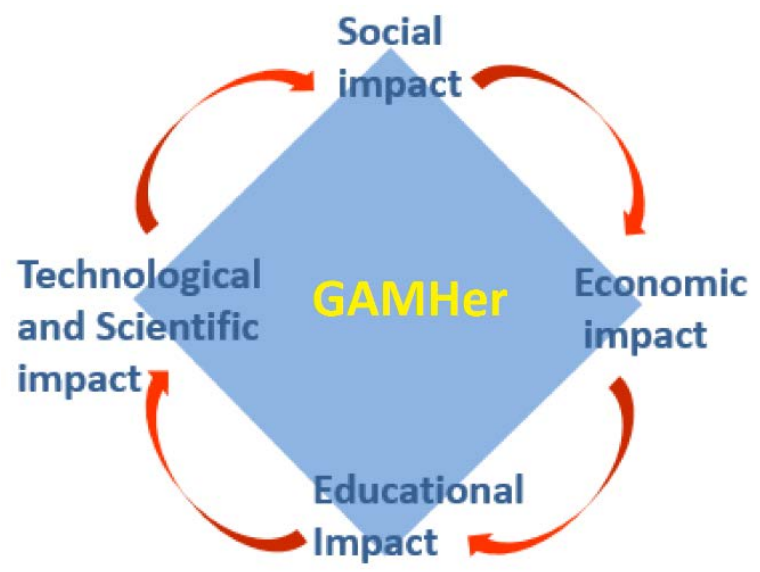

Figure 2. The impact at different levels of the GAMHer project.

GAMHer will provide a set of guidelines and services useful for a more cost effective data processing production workflow. They include:

- $\quad$ analysis of algorithms for automated data processing coming from photogrammetry and SfM, so that specific guidelines will ensure the production of accurate and reliable outputs and the procedures for validation for real and effective productive work;

- techniques and algorithms for as-built BIM generation from images and laser scans, that can speed up the generation of metric digital reconstruction while preserving the level of detail achievable from point clouds, in accordance to the requirements of the project;

- $\quad$ reuse of 3D digital reconstructions in an efficient data exchange for consecutive or transversal applications, avoiding the duplication of information towards a more productive and less dispersive approach. In this case, the reuse of meshes or BIM-based reconstructions will be exploited for FEM in a multiuser concept that stimulates collaboration between different specialists;

- $\quad$ integration of digital local data with GIS systems to fulfil the requirements of a multiscale \& multiLoD (Level of Detail) approach, with a particular attention to formats able to ensure interoperability among a variety of professional users;

- involvement of people at different levels, through the end user content validation starting from an expert user in the analysis of geospatial data, professional operators that already use geospatial data, and users with a limited experience in geospatial data.

In the next sections a synthetic description of the tasks and challenges that will be faced in the project are illustrated and discussed.

\section{APPLICATION IN MAINTENANCE, CONSERVATION AND ENHANCEMENT}

Following the themes addressed by the European directives (UEPPD), a particular attention is towards tools and guidelines for a data acquisition/processing workflow of images and laser scans in order to provide reliable and accurate 3D output for real and effective applications.

Historical Building Information Model (HBIM) is one of the technologies that are currently studied in the project. Data acquired with laser scanning and photogrammetry could be used, stored and managed along with other information in a HBIM. It clearly emerges the need to have some geometrical and radiometric data to support the modelling phase of the process, and some modern Geomatics techniques can offer a solid support for this purpose. Terrestrial Laser Scanner (TLS) and multi-view Structure from Motion (SfM) techniques can generate high fidelity point clouds, which represent the first step of the digital reconstruction phase (Remondino, 2011; Chiabrando et al., 2016).

\section{$3.1 \quad$ HBIM and FEM}

Another (correlated) environment where GAMHer is carrying out some assessments is related to the applications to structural analysis (e.g. FEM), to understand whether and how the 3D point clouds provided by photogrammetry or laser scanning can support faster and more automatic processes, and with what results in comparison with traditional approaches. Specific contributions will be provided especially in the integration and interface with FEM products, to be tested on selected historical buildings (see Fig. 3 for the example proposed in Bitelli et al., 2016, from the Bologna University research unit).

Actually, current software tools for transforming surveying data into structural models, typically finite element ones, still suffer from the inability to capture potentially critical details and a high degree of human interaction. Moreover, while point cloud data conversion into solid models is actively investigated (Hinks et al., 2013) especially for building facades, geometric survey and structural analysis of vaulted constructions has received scarce attention. In particular, recent papers on the subject (Ottoni et al., 2017) have shown that the use of digital surveying and modelling 
techniques reveals an underlying complexity, e.g., in evaluating the curvature of vaults and ribs. Furthermore, given the strong irregularity of the surveyed geometry, it is usually not possible to apply solid elements since unacceptable material incongruences are generated after the extrusion process. For these reasons, according to the modern paradigm of BIM objects, we shall exploit a parametric generation from point clouds of the vault shape that will allow structural analysis to be carried out by means of the Thrust Network formulation. It represents a simple and reliable strategy in which stresses within the vault are lumped to equilibrated compressive forces acting along the branches of a network.
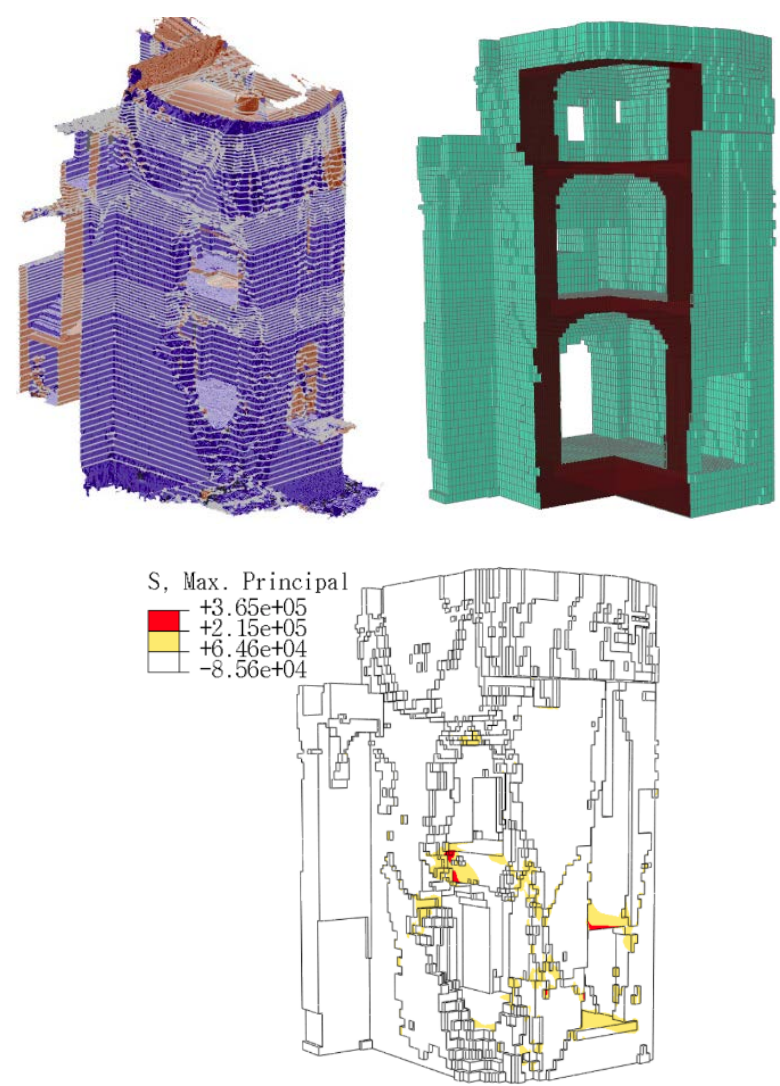

Figure 3. From point clouds to FEM using CLOUD2FEM procedure (Castellazzi et al., 2015) on a historical building: mesh with slices at variable distance (top left), voxel model (top

right) and one result of structural analysis (bottom).

Parametric modelling will be particularly useful to create 3D digital models for parts of the structure inaccessible to standard surveying techniques. Actually, no information about the thickness of the vault can be provided by point clouds since only the inner surface of the vault can be surveyed and destructive inspections are not always possible.

Integration of this information with additional data sources, such as historical reports, analysis on materials, non-destructive tests and infrared thermography will allow the user to dispose of an editable database containing the safety degree of the vault associated with several values of the vault thickness, since it can be automatically modified without redrawing.

\subsection{HBIM and GIS}

The project will also exploit, through a main contribution from Politecnico di Milano research unit, the integration and interoperability issues of BIM and GIS systems for combined surveying, management and planning projects. Both systems are still independently used in practical applications, whereas a more efficient and combined used is expected at European level to promote collaboration through integrated practices and open standards. It represents a fundamental step towards the creation of a common environment where the different specialists, involved in the project, can share and use geospatial information coupled with architectural/structural models, and vice versa.

The work will go through the available open standards for an interoperable BIM/GIS approach, as well as the technical operations which can be effectively carried out and those which require new specific functions. The geometrical survey from images and laser scans, that provide data at different levels (from the building until the cartographic scale), as well as the availability of existing data, will be taken into consideration for combined BIM/GIS projects. Particular attention will be paid to buildings that represent the largest part of commercial applications, where existing libraries are available only for specific objects with simple and regular geometry. This will require the development of procedures able to represent the geometric complexity captured by modern digital surveying techniques. The analysis will be then extended to large infrastructures that have a direct impact at the territorial scale, in which digital geospatial information has a primary importance. In this sense, the work will investigate an approach based on multiple levels of detail.

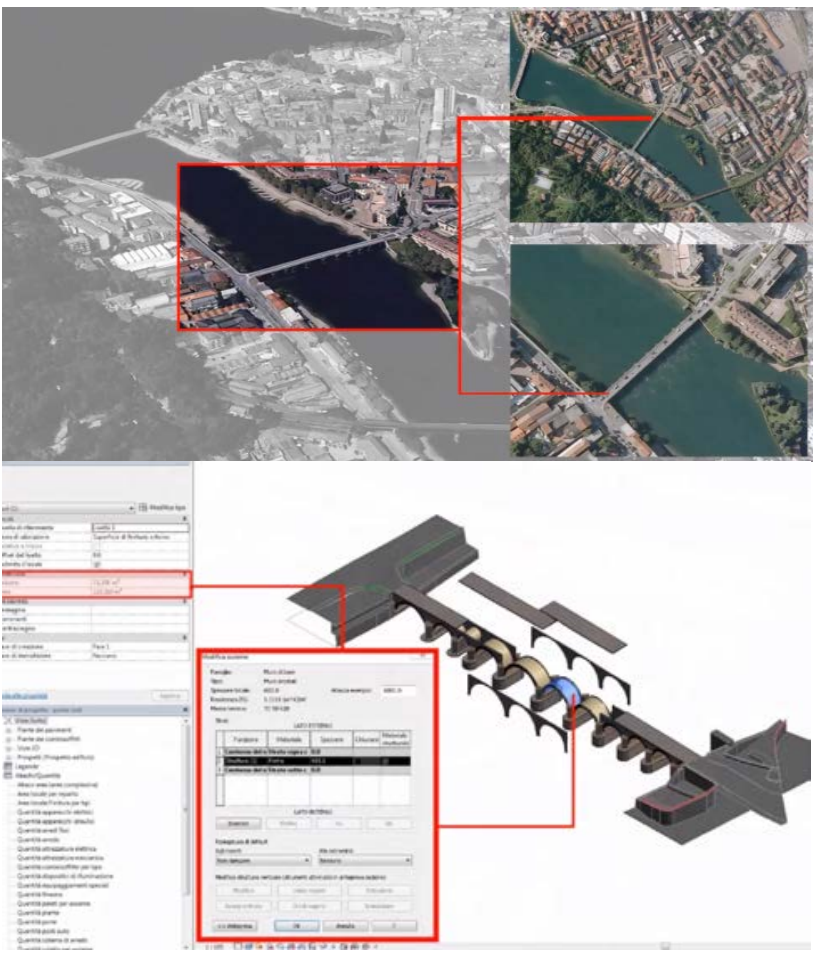

Figure 4. The integrated BIM-GIS concept for the mediaeval bridge Azzone Visconti, in Lecco (Italy).

\section{SHARING DIGITAL GEOSPATIAL DATA ON HERITAGE}

\subsection{Information for a wide user community}

The GAMHer project is aimed at both specialist and non-expert users, as highlighted in the overview section (2.1), thus implying also the involvement of the mass audience. Some crowdsourcing 
experiences will be proposed and tested on real cases of study, by taking the cue from already existing projects (for example, the reader is referred to http://archaeologydataservice.ac.uk/era/ and http://heritagetogether.org) in order to produce heritage data in conjunction with local communities.

\section{SHARING DIGITAL GEOSPATIAL DATA ON HERITAGE}

\subsection{Information for a wide user community}

The GAMHer project is aimed at both specialist and non-expert users, as highlighted in the overview section (2.1), thus implying also the involvement of the mass audience. Some crowdsourcing experiences will be proposed and tested on real cases of study, by taking the cue from already existing projects (for example, the reader is referred to http://archaeologydataservice.ac.uk/era/ and http://heritagetogether.org) in order to produce heritage data in conjunction with local communities.

A wide range of non-expert users can thus take part to the realization of collaborative photogrammetric projects, following the guidelines of simple protocols and workflows, presented on suitable web channels. This collaborative approach will allow one to manage and supervise the data produced from nonspecialists, to spread a basic education and good practices of acquisition, to sensitize and engage the public on their own territory and to provide a solution to the lack of funding for the heritage digitization.

The researches and the experimental tests, performed by other GAMHer partners, will help the University of Florence unit to define a correct methodology of geospatial data acquisition, suitable for different kind of instruments (from smartphones to professional cameras) and users, in order to obtain 3D models with a known accuracy and good quality. For this purpose, a simple protocol of specifications will be produced, by updating and improving the $3 \times 3$ rules already defined in the past by CIPAHD for digital photogrammetric software management, in order to obtain a final clear and easily communicable document. Some rules to acquire images for 3D modelling will be defined, such as: camera-object distance, lenses used, camera used, base/distance ratios, radiometric and geometric resolutions, basic metric information and so on.

Data from collaborative campaigns, gathered on a web portal, after a suitable process of evaluation and validation, could be processed or archived to make them finally available online as open data.

\subsection{D prototyping as a driver for knowledge sharing}

In the latest years, the development of 3D technologies applied to the field of Cultural Heritage $(\mathrm{CH})$ has led to results of utmost importance from the point of view of preservation, valorization, communication and fruition of our assets. This is confirmed by relevant projects, such as H2020-REFLECTIVE-7-2014: Advanced 3D modelling for accessing and understanding European cultural assets and Europeana. In particular, we experienced many interdisciplinary projects in which, thanks to the cooperation of different fields of research, incredible results have been obtained, through the technological collaboration of computer graphics, industrial engineering and experts in documentation, preservation and access of $\mathrm{CH}$.

Particular attention should be paid to the actual technologies in use for solid printing (digital fabrication) used for the realization of material copies, thus tangible, of three-dimensional digital virtual models (for more examples the reader is referred to Scopigno et al., 2015; Neumüller et al., 2014; Tucci et al., 2011; Balletti et al., 2016; 2017).
Even though ulterior developments to these technologies can be expected, the process of 3D printing has gradually gained levels of accuracy, which can nowadays be deemed as satisfying. This is even more true in the industrial field (from the manufacturing industry to the design industry), but also in other fields, such as the medical one (for example, for the realization of artificial limbs), and the $\mathrm{CH}$ field, which can benefit from new instruments for the restoration and preservation of cultural assets in museums. However, the widespread use of solid printers has highlighted the problem of building digital models that need to be printed and that of the conformity of the copy to the original.

The metric characteristics of precision and accuracy of the model printed with 3D technology are a typical problem of Geomatics, and have to be related with those of the digital model obtained through the survey analysis.

The question becomes very relevant when you consider projects such as that planned for the city of Palmyra, in Syria. The project, a very ambitious one, plans to recreate the ruins of the archaeological site by replacing them with their physical copies made through robotics and 3D printing. Projects like this make it obvious that there is the need to assess how much the printed model corresponds to the original. The physical model, which was traditionally used to anticipate and see what had not yet been realized, now becomes a true copy that will replace the original, becoming a monument itself.

To identify the different applications of such reproductions, it is now necessary to determine and verify metric accuracy of the digital and physical models: only in this way the copy will effectively replace the original in various fields, especially in the event that this must be preserved and its usability is limited to exposure. Physical models, in fact, are more and more frequently used where a physical contact is necessary; for example, museum exhibits devoted to children or the visually impaired.

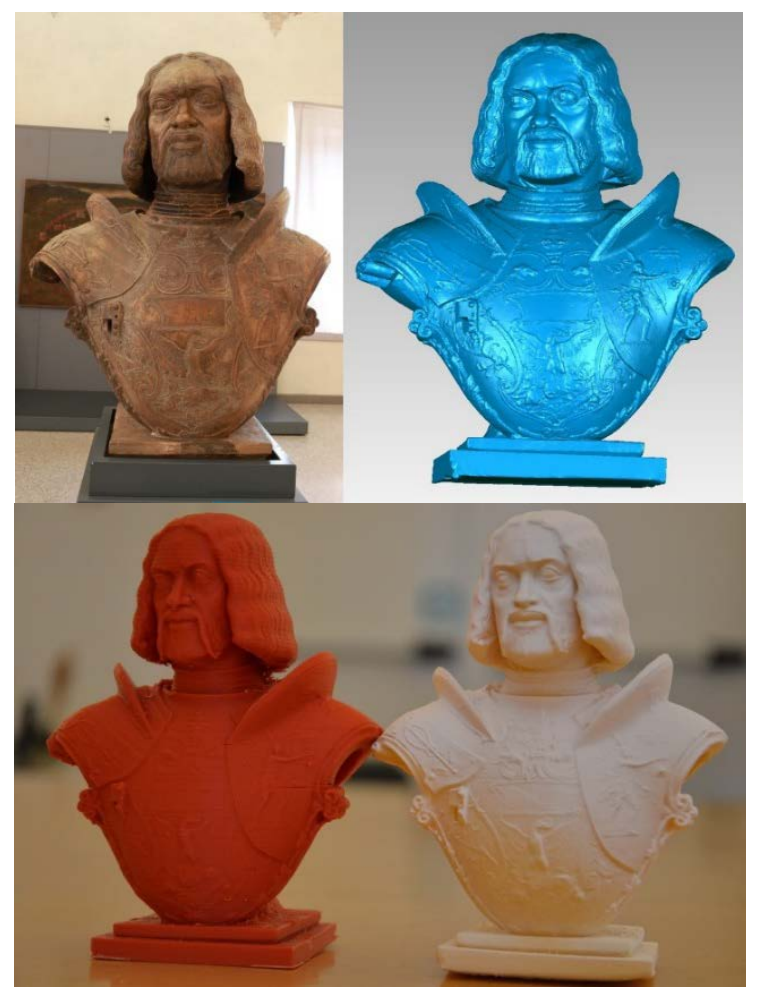

Figure 5. Digitization of the terracotta bust of Francesco II Gonzaga, in the City Museum of Mantua: the original bust, its digital reproduction and two physical copies. 
This contribution to the research project, which is mainly carried out at Università Iuav di Venezia, has the purpose to optimize the path that goes from the acquisition of a point cloud of an object (i.e. vase, statue, architecture, urban area) to its representation in a scaled 3D model (Figure 5). In this process one must of course take into consideration, on one hand, the accuracy of the acquisition tools (multi image photogrammetry, TOF laser scanner, triangulation laser scanner) and, on the other, that of the final output device: FDM, CNC, SLA, SLS printers, etc., to avoid diseconomies dictated by the different instrumental precision and resolution.

The main purpose is to analyze the metrical characteristics of the printed model in relation to the original object. Referring to the example in Figure 5, we want to focus on the methodology used: the acquisition in digital format of an artifact has given rise to two physical models, created by two different devices.

Both models have undergone a scan process and compared with the original. Since the object of the study was the precision that can be reached through these solid printing systems, the test was designed so to maintain unchanged conditions for the acquisition and processing of products as much as possible.

Thus, in the $\mathrm{CH}$ field there is the possibility of new systems of access, cataloguing and study, where the models, both virtual and tangible, represent the fundament of visualization and analysis of the form (also from the metric point of view) of each artefact of artistic and historical interest.

\subsection{Spreading of information via Web GIS}

Spreading a technology from niche sectors to a significant number of "expert users", and hence to a mass audience, exhibits interesting opportunities. It is a path similar to that which has transformed the traditional cartographic production towards neocartography and crowdmapping.

The on-line divulgation through Web GIS (O'Sullivan and Unwin, 2003) could be intended as the last phase of a complex process of collection and digital archiving of the built and/or historical archaeological heritage aimed to its valorization and tuition. In a Web GIS designed to spread information linked to buildings or cultural sites, the user accessing the platform is able to visualize services and products such as: positioning and area of the sites, spatial interaction with other places of interest (ex. UNESCO Heritage), territorial and environmental characteristics, satellite orthophotos, aerial or UAV mapping. GIS user-friendly software (Fig. 6 by D'Urso et al., 2017) allow one to automatically export the 3D models in HTML format, with an intuitive graphic interface, in which one can visualize the spatial coordinate of single points and all fields of the vector file. Each of these contents is described through the correct position and multimedia information as numerical values of measurements, processed images, photos, 3D models, monitoring data, diagrams, tables, additional technical information and links to external resources on the Internet.

In the GAMHer project, the University of Cassino and Southern Lazio will mainly develop such objective. One first aim is towards the interactive capacity of the typical user of a Web GIS to navigate into the geographical area of a building or the cultural site. Other functionalities are related to the choice of the data to be visualized, and those to be exported in compatible formats with the proper web platforms, as well as to be saved or to be printed the content.

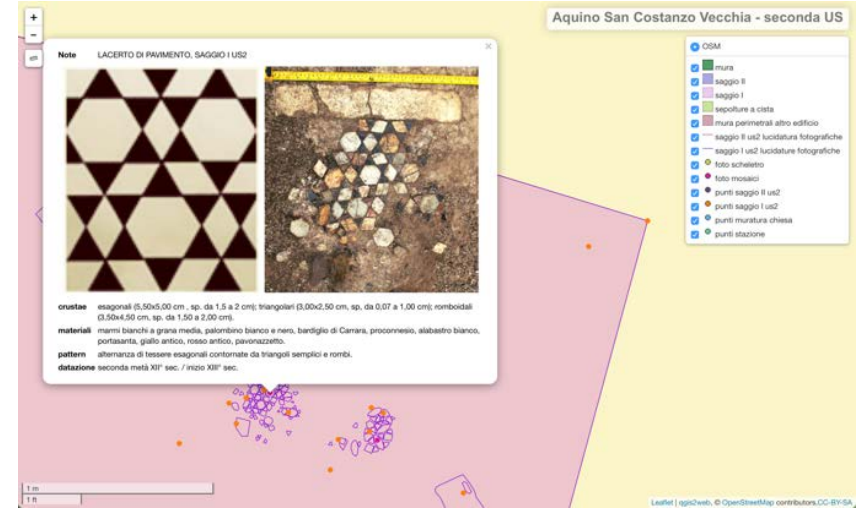

Figure 6. An example of a user friendly Q-GIS interface.

\section{TOWARDS BEST TECHNOLOGICAL PRACTICES AND QUALITY CERTIFICATION}

The need to realize comparative evaluations between modern 3D surveying techniques for the cultural heritage and, more generally, for the built environment, is transversal and common between the above mentioned contexts. The aim of the work carried out in GAMHer is to verify the quality of the algorithmic approaches and techniques available today, in both scientific and commercial products, privileging whenever possible open and low-cost software solutions since they are more easily transferable.

The theoretical and practical comparison between the traditional photogrammetry and SfM techniques is, undoubtedly, one of the themes more interesting and critic for a wider spread of these techniques. The modern trends in building up 3D models is to generate point clouds and to extract from them the necessary information to describe the space of the surveyed objects. Here, in the last 20 years the LiDAR techniques played a fundamental role for the change of surveying techniques: starting from the acquired point clouds, the expert operator does not select the needed points to describe the volumes of the objects during the survey. He extracts meaningful information after the automatic acquisition of the point cloud.

The growth of both radiometric and metric qualities of digital images allows digital photogrammetry to reach the same results of the LiDAR technique by using a set of oriented images (McGlone, 2013). The non-linear equations of collinearity need approximate values of unknowns and an iterative strategy to obtain the coordinates of the points and their precision parameter estimation (Gruen, 2003; Luhmann et al., 2014). At the same time, the Structure from Motion (SfM) approach can be used to extract metric information from images. It has led to a popular use of automatic software able to give (almost in real time) point clouds of an object imaged in a set of digital images. Calibration and orientation problems are solved by using alternative solutions based on projective geometry, which could result in point clouds without metric integrity. Apparently, the results seems the same in terms of point density but an effective metric comparison about the accuracy could reveal surprising results. 


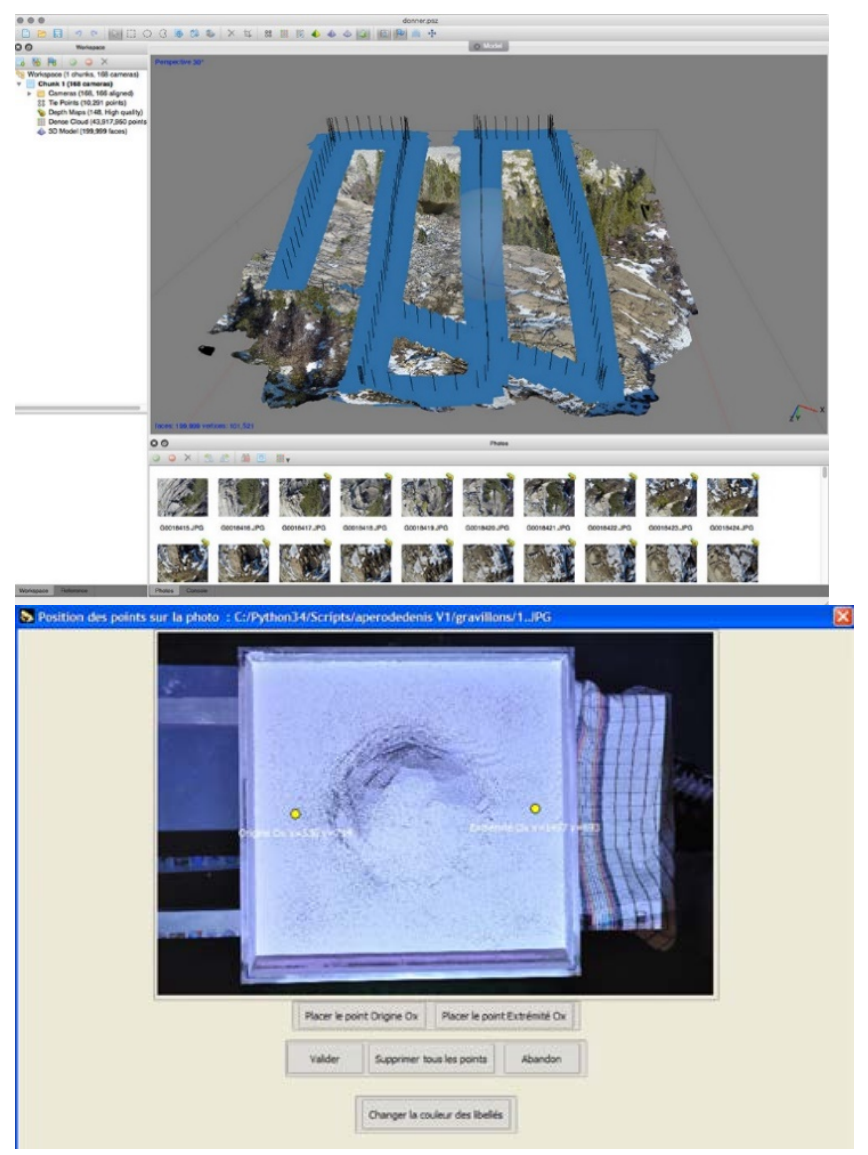

Figure 7. Photoscan, one of the most diffused SfM software and the user friendly Mic Mac interface: CEREMA.

The aim of the research in this specific topic is to compare, from a theoretical and experimental point of view, the two different approaches and possibly to put in evidence integration solutions of the two techniques. The first idea is to use the MicMac project (Fig. 7) to develop the research thanks the "open source" philosophy adopted by the developers. In the last months, a formal agreement between Politecnico di Torino and ENSG has been signed to develop new solutions able to generate point clouds and metric quality descriptors beside a new interface, which allow the user to understand and criticise the obtained results systematically.

If the expected results will be achieved, the research will offer a complete solution to generate the necessary point clouds for the subsequent 3D modelling and the quality parameters useful to provide an additional quality evaluation to 3D models.

Another promising study is towards the direct integration of data coming from photogrammetric surveys and 3D scanning devices, strengthening by a technique the results of the other without requiring necessarily a post-processing operation by examining the separate results.

The metric quality of the final 3D models, beside the other quality parameters (e,g, completeness, etc.), will allow a more correct management for the subsequent use in BIM generation.

\section{CONCLUSIONS AND OUTLOOKS}

This paper described the main activities that are being developed in the GAMHer project. The project actually started in 2017 with a fundamental consideration in mind: the heart of any conservation project of cultural heritage is an overall process of knowledge. To this end, accurate methodologies and sophisticated computational tools, both in the field of geometric survey and of structural mechanics, are currently available. Laser scanning and photogrammetry offer the ability to create point cloud models, digital mesh models and BIM to a millimeter level of accuracy. However, despite the high accuracy and automation level reached by both technology and software, rapid and reliable data processing and transfer still represents an open issue.

Specific topics of the investigation of GAMHer project are the use of 3D data as geometric database for GIS and BIM applications and FEM, test and definition of the best solutions to transform 3D point clouds generated by automatic Photogrammetry and/or SfM platform into 3D models for virtual and real replicas by means of 3D printers. Particular attention is paid to the solutions from classical Photogrammetry and Structure from Motion (SfM) (both from a theoretical and practical point of view), exploiting algorithms analysis and accuracy tests.

The methodology that GAMHer wants to use is based on a comprehensive study of recent and new experiences carried out by the different research units. GAMHer focuses on (i) the outline of the unsolved problems, (ii) the setting up of tests and experiments on selected case studies useful to underline the correct solutions, (iii) and the proposal of guidelines and best practices useful to offer a correct solution to the underlined problems. For these reasons, the outcomes of GAMHer are not intended to be used only by a community of Geomatics specialists, but also by a multitude of users that exploit images and laser scans in their activities.

\section{ACKNOWLEDGEMENTS}

This work has been carried out under the GAMHer project: Geomatics Data Acquisition and Management for Landscape and Built Heritage in a European Perspective, PRIN: Progetti di Ricerca di Rilevante Interesse Nazionale - Bando 2015, Prot. 2015HJLS7E.

\section{REFERENCES}

Balletti C., D'Agnano F., Guerra F., Vernier P. 2016. From point cloud to digital fabrication: a tangible reconstruction of $\mathrm{Ca}$ ' Venier dei Leoni, the Guggenheim Museum in Venice. ISPRS Ann. Photogramm. Remote Sens. Spatial Inf. Sci., III-5, 43-49, doi:10.5194/isprs-annals-III-5-43-2016.

Balletti C., Ballarin M., Guerra F., 2017. 3D printing: State of the art and future perspectives. Journal of Cultural Heritage. doi:10.1016/j.culher.2017.02.010.

Bitelli, G. Castellazzi, G. D'Altri, A.M. De Miranda, S. Lambertini, A. Selvaggi, I., 2017. Automated Voxel Model from Point Clouds for Structural Analysis of Cultural Heritage. ISPRS - International Archives of the Photogrammetry, Remote Sensing and Spatial Information Sciences, Volume XLI-B5, 2016, pp.191-197.

Castellazzi, G., D’Altri, A.M., Bitelli, G., Selvaggi I., Lambertini A., 2015. From Laser Scanning to Finite Element Analysis of Complex Buildings by Using a Semi-Automatic Procedure, Sensors, 15, 18360-18380, doi:10.3390/s150818360.

Chiabrando, F., Sammartano, G., Spanò A., 2016. Historical buildings Models and their Handling via 3D Survey: from Point Clouds to User-Oriented HBIM. The International Archives of 
the Photogrammetry, Remote Sensing and Spatial Information Sciences, Volume XLI-B5, pp. 633-640.

D'Urso, M. G., Corsi, E., Nemeti, S., Germani M., 2017. From excavations to web: a GIS for archaeology. The International Archives of the Photogrammetry, Remote Sensing and Spatial Information Sciences, Volume XLII-5/W1, 2017, GEOMATICS \& RESTORATION - Conservation of Cultural Heritage in the Digital Era, 22-24 May 2017, Florence, Italy

Hartley, R., Zisserman, A., 2003. Multiple view geometry in computer vision. Cambridge University Press.

Hinks T., Carr H., Truong-Hong L,, Laefer D.F., 2013. Point Cloud Data Conversion into Solid Models via Point-Based Voxelization, J. Surv. Eng., 139:72-83.

Gruen, A., and Huang, T.S., 2013. Calibration and orientation of cameras in computer vision. Vol. 34. Springer Science \& Business Media.

Luhmann, T., et al., 2014. Close-range photogrammetry and 3D imaging. Walter de Gruyter.

McGlone, J. Chris, 2013. Manual of photogrammetry. American Soc. for Photogrammetry and Remote Sensing.

Neumüller, M., Reichinger, A., Rist, F., Kern, C., 2014. 3D printing for cultural heritage: preservation, accessibility, research and education. In: Ioannides M, Quak E, editors. 3D research challenges in cultural heritage: a roadmao in digital heritage preservation. Heidelberg: Springer; p. 119-34.

Ottoni F., Freddi F., Zerbi A., 2017. From “models” to reality and return. Some reflections on the interaction between survey and interpretative methods for built heritage conservation. The International Archives of the Photogrammetry, Remote Sensing and Spatial Information Sciences, Volume XLII-5/W1, 2017 GEOMATICS \& RESTORATION - Conservation of Cultural Heritage in the Digital Era, 22-24 May 2017, Florence, Italy.

O'Sullivan, D., and Unwin, D., 2003 Geographical Information Analysis John Wiley \& Sons, Inc. Hoboken, New Jersey.

Remondino, F., 2011. Heritage Recording and 3D Modeling with Photogrammetry and 3D Scanning. Remote Sensing, Vol. 3(6), pp.1104-1138.

Scopigno R., Cignoni P., Pietroni N., Callieri M., Dellepiane M., 2015. Digital Fabrication Techniques for Cultural Heritage: A Survey. Computer Graphics Forum. doi:10.1111/cgf.12781

Tucci G., Bonora V., 2011. From real to "real”. A review of geomatic and rapid prototyping techniques for solid modelling in cultural heritage field. Int. Arch. Photogramm. Remote Sens. Spatial Inf. Sci., XXXVIII-5/W16, 575-582, doi:10.5194/isprsarchives-XXXVIII-5-W16-575-2011. 